\title{
Greening of IP-Based Video Distribution Networks: Developments and Challenges
}

\author{
Adrian Popescu \\ Department of Communication Systems \\ Faculty of Computing \\ Blekinge Institute of Technology \\ 37179 Karlskrona, Sweden \\ adrian.popescu@bth.se
}

\begin{abstract}
-
The creation and the distribution of video content is a multistage process that refers to the aquisition of video source, content production and packaging, and distribution to customers. The major components are the access networks, metro/edge networks, core networks, data centers and storage networks.

Today, the access networks, of type wireless and wired, dominate the power consumption of the chain. However, it is expected that, with increasing access speeds, the core network routing will dominate the power consumption of the chain as well. Furthermore, it is also expected that the power consumption of Data Centers (DCs) and Content Distribution Networks (CDNs) will be dominated by the power consumption of data storage for content that is infrequently downloaded as well as by the transport of data for content that is frequently downloaded.

The paper provides an overview of the problems related to the greening of IP-based video distribution, with particular focus on recent developments and the associated challenges. These are research topics planned to be solved by the last call CelticPlus project proposal CONVINcE (Consumption OptimizatioN in Video Networks). This research project has received the EUREKA Celtic-Plus label for funding approval in five European countries: France, Sweden, Finland, Romania and Turkey. It is considered to be a very high quality research and the topic is considered to be very relevant to our future from ecological and economical view.
\end{abstract}

\section{INTRODUCTION}

Video Distribution Systems (VDS) are basically a set of audio and video devices, peripherals and equipment for interfacing, switching, routing and distributing of video and picture signals sourced from multiple devices and distributed to geographically dispersed multiple devices. VDS are expected to be able to provide distribution services of different categories like many to one, one to many and many to many. For doing this, a set of specifications and design guidelines are used.

Technical solutions with business value indicate today a good mixture of performance, repeatability and easy-to-use facilities. Video distribution has almost completely moved from switched networks to IP networks, also combined with the use of IP-capable endpoints and the use of H.323 to offer extensive functionality for video. At the same time, the enterprise Voice over IP (VoIP) environment continues the massive transition to Session Initiation Protocol (SIP) for functions like call setup, management and termination of multimedia sessions, also requiring internetworking with H.323 based video endpoints.

The transition from switched networks to IP has today yielded significant improvements in terms of quality and performance but at the price of increasing the energy consumption. This is because the video delivery is associated with several serious challenges, the solutions of which demand for more energy. The video is a bandwidth hungry application, the streaming of video demands for real-time consistent network performance, sophisticated distribution schemes as well as that the video is perceived by the end user as a continuous longterm experience. All together, this poses an increasing demand for developing solutions for IP-based video distribution networks that are energy-efficient as well. The goal of the paper is to report an overview of the main elements of video distribution networks together with several particular solutions for energy saving and the challenges related to them.

The rest of the paper is as follows. Section II gives a short presentation of the high level network structure for video distribution. Section III is about greening of Radio Access Networks (RANs). Section IV presents the main research directions for the greening of core networks. Section $\mathrm{V}$ is about network virtualization and the challenges related to this. Section VI is about content distribution and Data Centers (DCs) as well as the challenges related to their greening. Section VII presents the Cognitive Network Operation and Management (CNOM), which is an important element in the future greened video distribution networks. Finally, the paper is concluded in Section VIII

\section{VIDEO OVER IP Distribution SYSTEMS}

The creation of video content and the distribution over IP is a sophisticated process that follows a chain model from the acquisition at the video source, through the production and packaging of the content to the final element of distribution to viewers [1]. This means that a video distribution system contains several parts, which are the contribution, the primary distribution and the secondary distribution.

Video contribution refers to functions like capturing and initial processing of the video content as well as the initial transport prior to distribution. For instance, the video content 
may be captured at a football stadium and it may be transported to a central broadcast network facility, which is placed in another location. The video streams used in this phase may be compressed or uncompressed, depending upon the particular existing situation in terms of bandwidth availability and the particular demands for quality.

The distribution video is sent from the central broadcast facility to other broadcast facilities for ultimate transmission to end users. Distribution configurations of type one to one (unicast) and one to many (multicast) are typically used in this case.

There are two categories of distribution systems, the primary distribution and the secondary distribution [1]. The primary video distribution providers are responsible for the transport of video content from the production entity to the secondary distribution entity for ultimate transmission to end users. Primary distribution services are normally using compressed video formats, e.g., MPEG-2 or MPEG-4 or JPEG 2000.

The secondary video distribution providers manage the transport of video content from the primary video distribution providers to the end consumers. Examples of secondary distribution services are IPTV and cable TV. Secondary distribution services are normally compressed with MPEG-2 or MPEG-4 standards, with rates of 2-4 Mbps for Standard Definition (SD) systems and 8-20 Mbps for High Definition (HD) systems. The distribution configurations are of type one to one (unicast) and one to many (multicast).

Basic operations done on the video signals along the video distribution chain are video coding and compression, encapsulation, forward error correction, transmission, reception and decapsulation, error correction and decompression.

Like other categories of service provider networks, video over IP distribution systems are expected to provide services with a good mix of simplicity, scalability, security, manageability and cost effectiveness. Service Level Agreement (SLA) requirements for video are about parameters like network delay, network jitter, packet loss, availability, loss recovery.

The requirements for the greening of IP-based video distribution networks heavily complicates the picture. That means one needs to take a closer look at the component elements in the primary and secondary distribution networks and to analyze the greening mechanisms used in the particular cases. The major components are the access networks (with different categories of technologies of type wired and wireless), the core networks, the Data Centers (DCs) and the storage networks used to provide Web-based services like IPTv, content distribution and cloud-based services.

\section{RADIO ACCESS NeTwORKS}

One of the most serious challenges existing today is the huge increase in demand for mobile data traffic [2]. This is a multi-dimensional problem, which refers to the dramatic increase of the mobile traffic, proliferation of new mobile services, change of the characteristics of wireless data (most of data comes today from video services and it has been also observed that at least $50 \%$ of wireless data comes from/to smartphones via $\mathrm{Wi}-\mathrm{Fi}$ and less than $50 \%$ via the cellular networks), ineffective spectrum usage combined with nonoptimal use of radio resources and also the multiplication of interference problems.

Related to this, we have the problem of continuous increase of energy consumption due to the development and deployment of Base Stations (BS). This problem is exacerbated because of the low energy efficiency associated with a BS. For instance, a $3 \mathrm{G}$ system with 12 transceivers consumes $3802 \mathrm{~W}$ of AC power supply as the feeding power [2]. Every transceiver has an input power of $200 \mathrm{~W}, 60 \mathrm{~W}$ of which are consumed at idle time and the rest of $140 \mathrm{~W}$ are consumed for transmission. The output power after a Power Amplifier (PA) is of $40 \mathrm{~W}$ and the final power sent over the air is $10 \mathrm{~W}$ only. That means PAs consume $70 \%$ of the total power, with an overall efficiency of $28 \%$. On the other hand, the overall efficiency of the BS is only $3 \%$. For $3 \mathrm{G}$ BS the overall efficiency of PA can however be $45 \%$ and the overall efficiency of the BS is less than $12 \%$ [2], [3]. As an example, table 1 shows a list of transmission power (at $10 \mathrm{Mhz}$ bandwidth) expressed in $\mathrm{dBm}$ (i.e., referenced to 1 milliwatt) for different cell sizes together with the backhaul characteristics [4].

TABLE I

TRANSMISSION POWER FOR DIFFERENT CELL CATEGORIES [4]

\begin{tabular}{lll}
\hline Cell category & Transmission power & Backhaul \\
\hline \hline & $(10 \mathrm{Mhz}$ bandwidth) & (HetNets) \\
\hline \hline Macrocell & $46 \mathrm{dBm}$ & S1 \\
\hline Remote Radio Head & $24-30 \mathrm{dBm}$ & Optical fiber \\
\hline Relay & $30 \mathrm{dBm}$ & Wireless \\
\hline Picocell & $24-30 \mathrm{dBm}$ & X2 \\
\hline Eemtocell & $20 \mathrm{dBm}$ & IP (Broadband) \\
\hline
\end{tabular}

An important observation is that the large amount of energy consumed and wasted in Radio Access Networks (RANs) also increases the operational expenditure of the systems. It has been for instance observed that these networks are responsible for about $90 \%$ of the operating expenditures (OPEX) of network operators [5]. This problem is expected to become even more serious in the future, when the mobile operators face important challenges in form of raising OPEX (due to continuous upgrade of services and increase of energy costs) combined with dropping average revenue per user (ARPU) due to significant price reduction and increased competition.

Furthermore, the fact that the traffic generated by RANs has been observed to be uneven ( $10 \%$ of the sites carry about $50 \%$ of the traffic [5]) is an indication that there is room for better traffic distribution and energy savings. Better architectures can be developed for energy-efficient Base Stations, e.g., by using virtualization techniques or by letting lightly loaded Base Stations change the status to sleep state. The signal processing part can be moved from the Base Stations into the cloud, and we can, e.g., develop energy-efficient protocols, middleware and other diverse smart algorithms able to provide both power 
savings and performance provision.

Another important development is the appearance of heterogeneous networks. Whereas the macrocells and their deployment have been shown to be efficient in providing the coverage and capacity needed for voice and low data rate services, heterogeneous networks are more efficient for higher data rates and more advanced wireless services. The appearance of small cells (pico- and femtocells), particularly in the urban areas, has changed the premises. The operators have now the possibility to cover high data traffic areas such as shopping malls or offices or even households without the need to setup a mobile mast or tower. The better mobile coverage offers the possibility to meet the increased demand for mobile data in smartphones and tablets.

A much denser architecture, based on smaller cells, is expected to support the increasing demand for high data rates. In the new architecture, several important trade-offs need however to be re-evaluated. These are the energy efficiency, network performance, spectrum and deployment efficiencies [6]. The evolution from macrocells to small cells (microcells, picocells and femtocells) enables high data rates to multiple users by increasing the energy efficiency resulting from the decrease in propagation distance. This in turn has the positive effect of reduced transmission power and, potentially, a more complex modulation scheme leading to even higher date rates. The so-called cooperative heterogeneous networks, with the help of which better performance can be obtained, are very promising in the provision of advantages like higher spatial diversity, higher throughput, lower transmitted power, lower delay and good adaptability to network conditions [6], [7].

At the same time, decreasing the cell sizes has the negative effect that the number of handovers is increased as well as the level of interference and complexity of deployment for the resulting network. Additional difficulties are because site acquisition for Base Stations is very difficult in urban areas due to objecting citizens and homeowners. Other problems connected with heterogeneous networks that need to be solved are about interference mitigation, performance provision, handover, self-organization, resource allocation [8]. These problems are further exacerbated because of spectrum limitations due to rigid regulatory rules existing today for spectrum allocation and access. The problem of spectrum limitation can in turn be solved with the help of new radio technologies like Cognitive Radio Networks (CRNs) [6].

\section{Core Networks}

There are several important things that need to be considered in the design of core/metro networks for video distribution. These are the fact that video is bandwidth hungry application, which requires real-time consistent network performance (e.g., demand for maximum delay of $150 \mathrm{~ms}$ for video chat to ensure lip-synchronization) and also that it is perceived by the user to be a continuous long-term experience. Other important elements are the needs for transcoding and for the so-called Backend as a Service (BaaS) facility to support the backend capabilities (e.g., servers, applications, databases) in building fully featured mobile applications by supporting an applications user-facing front end [9].

A good practice is to first have a close look at the whole chain for video distribution and to understand where the bottlenecks happen. Furthermore, the video network should be able to operate and to adapt (at more time scales and layers) based on the perceptual distorsion in the video stream as well.

As a general comment, the existing policies used today in wide area networking are totally in opposition with green networking objectives. These are the over-provisioning and the redundancy policies. The goal of these policies is to provide facilities for Quality of Service (QoS) support, but this is done at a high price in form of energy waste. The conclusion therefore is that there is a strong need for reviewing these policies as well as for the development of green policies for wide area networks.

There are four directions to follow for the greening of wide area networking, which stem from observations done on the root causes of energy waste. These are as follows [5], [10]: adaptive link rate (where the link rate is adapted either by turning off links during idle time periods, the so-called sleeping mode, or by reducing the link rate during low utilization period), interface proxying (delegate traffic processing to more energy efficient entities, e.g., cloud solution), energy-aware infrastructure (this is mainly about energy-aware routing, based on collaboration among individual entities; other concepts are re-engineering, sleeping and dynamic adaptation) and energyaware software and applications (where the operating system and the applications are modified to participate in the reduction of energy budget. It is important that we adopt a multidimensional view in the sense that we estimate the effects of the algorithms in the following domains: energy consumption, other environment elements, economy aspects, regulatory and engineering elements [5].

Finally, an important part is regarding the study and development of energy-aware routing algorithms. Generally, such algorithms demand for the development and implementation of off-line strategies with the consequence of deep modifications needed to be done in IP routing [10]-[12]. One of the most important research challenges is therefore the development of energy-aware routing algorithms that are compatible with the existing interior and exterior routing algorithms (e.g., OSPF, BGP). Other important elements are about determination of network power consumption model, throughput-guaranteed power-aware routing model, and determination of appropriated green metrics, able to capture the greening properties of routing in wide-area network, together with appropriated measurement methodologies to capture the these properties.

\section{Network Virtualization}

Network virtualization means that a set of mechanisms are used to let more services operate on a given set of heterogeneous resources with positive effects like more efficient delivery of services, reduced resource costs, reduced energy, 
reduced cooling costs, improved energy management, reduced carbon footprint, multi-tenancy, reduced CAPEX and OPEX. Examples of resources are hardware, servers, network links, storage resources, software resources, Base Stations (BS). The resources can be reserved on an on-demand basis and the users do not need to have knowledge about the physical resources.

Basically, network virtualization is used to isolate parts of networks and computers and to group these resources by using virtualization techniques to create virtual networks [13] In other words, this is not about virtualizing connectivity but about virtualizing the infrastructure as a whole. The network services are decoupled from the physical network hardware. We have in this case a so-called meta-architecture, which means that multiple different networks are concurrently accommodated. Design goals regard scalability, manageability, flexibility, isolation, security and programmability.

There are several solutions for the management of Network Virtualization Environments (NVEs) [14]. These are depending on the management target (node management; link management; network management), management function (provisioning; monitoring; interfacing), management approach (centralized and distributed management; autonomic and policy-based management).

Although virtualization is today a mature research field, virtualization platforms designed explicitly to reduce the energy consumption in IP-based video distribution networks are still focus of intense research activity [13], [15]. Important challenges are regarding interoperability (execution of virtual appliances in different datacenter environments), isolation (security isolation, performance isolation), aggregation (aggregating multiple resources into a single resource that is offered to end user), performance trade-off (minimizing the performance penalty when using different industry hardware standards), management and orchestration (demands for a consistent management and orchestration architecture), scalability (maximizing the number of virtual network supported over a set of physical resources) [16]-[18].

Two other important challenges are about increasing the level of flexibility, transparency and optimization for delivering the service and the research and development of green virtualization platforms. The virtualization platform must be homogeneously managed and controlled irrespective of the type of infrastructure. Eventual problems related to the category of resource, network segmentation and administrative partitioning must be overcome and the expected flexibility and transparency be provided. For solving these challenges, the concept of cognitive network operation and management needs to be used.

Recent developments like Self-Organizing Networks (SON), Software Defined Networking (SDN) and the associated OpenFlow have advanced the idea of more local and autonomous network management [17], [19]-[21]. This is an architecture where the network control functionality is separated from the forwarding functionality. In other words, the control is separated from hardware and a software application called controller takes over the control function.

There are three layers in the Software Defined Networking (SDN) architecture [17]. These are the network infrastructure (contains the SDN enabled network equipment, e.g., OpenFlow switch and router), the control layer (contains the SDN control software to provide different networking services, also including the middleware) and the application layer (contains different business logics).

Management operations can, e.g., be classified into management of infrastructure providers and management of service providers. The infrastructure manager controls the physical nodes and the virtual nodes are hosted on physical nodes. Every physical node has a hypervisor that allows the creation and the management of virtual nodes. In a similar way, virtual links can be created with the help of agents placed in the physical nodes. The virtual links can be created, e.g., by configuring Ethernet VLANs, MPLS and IP routers. The network traffic is controlled by creating forwarding-table rules in every device like routers, switches.

Given the complexity of the end-to-end (e2e) chain in an IP-based video distribution network, with several categories of networks involved, non-centrally controlled SDN models need to be investigated as well. The goal is to obtain the benefits of SDN and OpenFlow without using massive control to control the connections and the traffic. The Internet Engineering Task Force (IETF) is considering a number of solutions and changes to existing protocols like Border Gateway Protocol (BGP) to allow current IP networks offer SDN-compatible features without relying on controlling individual forwarding tables in every switch and router [22]. One needs in this case to study and to compare the energy consumption and savings for the two alternative solutions, i.e., centrally controlled SDN model and non-centrally controlled SDN model. The parameters used in this study should be energy consumption, configuration limitations, software programmability, workload mobility, multitenancy limitations, network service limitations, network scale limitations, network interoperability.

Another important research item is regarding the extension of SDN and the associated OpenFlow to wireless networks, with particular focus on $4 \mathrm{G}$ and Wi-Fi. Today, there are a few solutions existing for this, like the results reported by Huawei [23]. The goal in this case is to do research and to develop software-defined mobile networks (SDMNs), which are similar to SDN but able to provide network virtualization for wireless networks [23]. Another important goal is to study the performance of this, and to compare it to other solutions.

\section{Vi. Content Distribution and Data Centers}

Content Delivery Network (CDN) is basically a ring of content storage points located near access providers to ensure better and cheaper content experience. The content is replicated over the mirrored servers appropriately placed in Data Centers (DCs) at various locations. Requests for content are typically directed to surrogate servers that are optimal in some way. Media delivery can accordingly be done with reference 
to different parameters used in the optimization process like minimum cost, highest possible bandwidth, lowest number of hops, lowest delay, minimum power.

CDN consists of four layers: Basic Fabric (to provide the infrastructural resources like operating systems, distributed file systems, content indexing system); Communication and Connectivity (provides the core Internet protocols and the CDN specific Internet protocols, e.g., overlay protocols, security layer); $\mathrm{CDN}$ layer (provides core $\mathrm{CDN}$ functionalities like CDN services, CDN types and content types); and End-users (Web users, Web browsers) [24].

The functional components of CDN are content distribution (moving the content from the source towards users), request routing (selecting the best location for retrieving the requested content), content routing (delivering the content from the most appropriate place to the client requesting it), content composition and processing (creating and adapting the content to user preferences and device capabilities) as well as authorization, authentication and accounting (enabling the monitoring, logging, accounting and billing of content usage). An analysis of the energy consumption in CDNs means that we need to closely look at these functional components.

Perhaps the most important component in the CDN architecture that influences the energy consumption and also the associated business model is the CDN type. Towards this goal, there are several CDN categories, namely Public CDN, Enterprise CDN, Edge Services, and P2P File Sharing. Information about the performance of these categories of $\mathrm{CDN}$ is provided in [24].

Over the last years, the development in content delivery has undergone a fundamental shift, from the existing devicecentric (middle-box) model to a more efficient approach, a cloud-based solution, the so-called edge cloud [25]. This is a solution where the intelligence is moved away from the network core toward the network edge as well as that the storage and some functionality are moved away from the home toward the network edge [26]. The main advantage is that this follows the development we have today, where the innovation takes place at the network edge, more computing and storage power in users devices as well as fewer legacies. Another important advantage is that in this architecture the processing is done closer to the user, and this gives low latency for content delivery and enhanced subscribers Quality of Experience (QoE). Further, this also means that the user has increased facilities to control sources of content in a more efficient way, while avoiding increasing the terminal load. The core network is expected to become stateless and the edge networks will be only stateful elements of the networks. Application scenarios can in this case be network portability, network federation and network partitioning.

On the other hand, the drawback in this case is the energy increase associated with this architecture, at the level of the whole network. This is because of the energy needed to support the multiple edge entities (e.g., Data Centers) as well as the increased processing power needed in the mobile terminals. Furthermore, the complexity of developing modern mobile applications means that the mobile applications need support, e.g., in the form of backend services, for purposes like user management, data storage, push notifications and social network integration. The mobile cloud backend services are a specialized form of Platform as a Service (PaaS) used to support the development of mobile applications.

Generally, there is a list of the so-called best practices, which is useful for building of modern (Web) applications for mobile devices and for improving the QoE like, e.g., elimination of HTTP requests and round trips, use of compression, management of JavaScript parse time, avoidance of layout and style calculation, remove duplicate scripts, preloading of different components, avoidance of redirects, image optimization, reducing the DNS lookups, minify JavaScript [27]. These measures involve, many of them, energy increase. This is a problem that needs to be investigated in order to save energy in mobile devices and the edge entities. In other words, one needs to investigate what is the best compromise with reference to both providing QoE and energy saving.

It is also important to consider the developments imposed by the 3rd Generation Partnership Project (3GPP), which are adopting new standards in the form of Release 12 LTE Advanced and, connected with this, new mechanisms for content and network optimization techniques to provide acceleration of content delivery [28]. For instance, an interesting solution has been suggested by Ericsson and Akamai in the form of mobile cloud accelerator, which reserves a portion of the network bandwidth for the premium content [28]. The advantage is that the content with higher priority can be provided better transport service, with less performance deterioration due to network congestion.

There are three categories of accelerated content delivery in mobile networks [28]. They are based on mobile system evolution (the goal is to reduce the traffic volume and accordingly the network congestion; techniques contain caching, data redundancy elimination, prefetching and data compression), content and network optimization (handover optimization, queue management, network coding, TCP optimization, session layer optimization) and mobile data offloading (cross domain techniques such as content and protocol adaptation). Each of these alternative solutions contains several methods to accelerate the content delivery, each of them with own advantages and drawbacks. There is therefore need to evaluate these mechanisms with regard to energy saving as well.

Several important consequences of this development are that carriers deploy multiple delivery platforms with increased power consumption. There is therefore need to develop virtualized Data Centers (DCs) in order to save money and energy as well as to develop new business models to capture the new situation. Network operators are expected to follow this development and to develop better business models. These models are of course expected to catch the multiple roles of network operators like, e.g., the case when network operators also play the role of infrastructure providers of edge networks. 
Another important research is regarding the virtualization of Edge Data Centers (EDCs) as a way to improve the energy efficiency at the level of whole video distribution network [29]. This solution is of type multi-tiered, where EDCs complement the remote DC to provide high quality distribution services at low cost. Virtualization of EDCs is expected to provide advantages like better management flexibility, better resource utilization (not only energy), lower cost, scalability. On the other hand, this solution has a number of drawbacks that must be investigated and reduced, e.g., increased risk for security attacks, less performance isolation, limited management flexibility. Other research challenges are regarding addressing schemes, efficient mapping of virtual resources to physical resources, scalability, performance isolation, interfacing, security, pricing [29].

Basically, a virtual DC (vDC) is a DC where (parts of) hardware are virtualized like servers, routers, switches, links. Hence, a vDC is composed of different types of virtual nodes (e.g., Virtual Machines VMs, virtual switches, virtual routers), each of them being allocated some particular resources (in the form of CPU, memory, disk space). Furthermore, we also have virtual links, which are characterized by bandwidth and eventually other parameters like propagation delay, error rate. A special software (or firmware) called hypervisor is used to divide the equipment into multiple isolated and independent virtual instances. Based on this, Virtual Networks (VNs) can be created as being sets of virtual networking resources.

The research efforts on EDC need to be focused on several directions. These are regarding the study and the design of green VDC, where different algorithms take into account the energy consumption and accordingly adapt the performance to a certain (given) level of energy saving. Furthermore, there is need to study and to develop appropriated business models, able to capture the new mechanisms for content distribution. These models should consider (as variables) the participating players, which are end-users, Infrastructure Provider (InP) and Service Providers/tenants (SP).

\section{Cognitive Network Operation AND MANAGEMENT}

An important part in video distribution networks is regarding the development of elements for self-managing and reconfigurable networks. This is about what is called Cognitive Network Operation and Management (CNOM) system, which extends the concept of 3GPP Self-Organizing Networks (SON) to radio access and wide area networks operation [19]. The goal is to develop an efficient combination of legacy wireless network management with adapted exploratory results in the field of autonomic systems.

CNOM provides facilities for the efficient increase of resource usage, energy saving, cost-efficient network operation, reduction of overall costs associated with network capital, operation and administration. At the same time, CNOM is also about adaptive mapping of user requirements, preferences and context onto the offered services by considering the resource assignments, strategies and other policies of the service provider [13]. This demands for real-time monitoring and control of the network and associated resources.

Connected with this, an important element is the need to develop the so-called delivery-centric paradigm, to replace the existing content distribution paradigm. This is necessary because today it is difficult to optimize the content access using static design-time decisions, to follow the environmental variance [30]. The existing content-centric networking API means that the applications are allowed to issue content requests agnostic to location or protocol, where high-level requests in form of, e.g., performance, security level are stipulated. The problem is because of the complexity of this model with the consequence of limited performance. On the other hand, the solution of delivery-centric paradigm is based on considering the environmental variance (e.g., consumer HTTP variance, temporal HTTP variance, consumer BitTorrent variance, temporal BitTorrent variance) to easy up the content delivery. By doing this, the ability to adapt to the networking environment is increased and accordingly the facilities to satisfy the customer requirements and to develop green networking solutions for media delivery.

The network has the ability to observe and to learn from the environment and accordingly to react in response to different conditions or events based on prior acquired knowledge and an adequate reasoning algorithm. For doing this, the network is enhanced with knowledge representation, automated reasoning and learning facilities. At the same time, one needs to provide elements for adaptive mapping of user requirements, preferences and context onto the offered services by considering the resource assignments, strategies and other policies of the service providers.

There are two categories of software suite, used for the CNOM of access network and for the CNOM of core network, respectively. Basically, these software suites are similar, except certain functions implemented in overlays, which are different at access networks and at core network. For instance, a specific decision support system can be developed that is suitable for access networks and for core networks, respectively.

An example of network architecture based on using CNOM is suggested in [31]. This is a composite wireless communication network, which has several Radio Access Networks (RANs), each of them with at least one Radio Access Technology (RAT). Some RATs may share a common frequency band. A so-called Cognitive Network Manager (CNM) is used to control the multi-domain network. This is an entity that collects information about the existent resources in multiple domains and, based on some particular decision making algorithm, takes decisions needed for the particular situations. In a similar way, a so-called Cognitive Terminal Manager (CTM) is used for similar purposes in terminals (UEs). Furthermore, a so-called Common Control Channel (CCC) can be used for control purposes, to provide the signaling needs among the participating entities in different networks (cross-network signaling). To avoid interference with the licensed bands, the 
CCC must be placed in out-of-band frequencies like, e.g., the unused parts of the UHF band recently released by the discontinuation of analog TV. The main advantage is that the control data is separated from the user data transmission, and the synchronization problems are eliminated.

A reconfigurable software suite with three layers can be used in both CNM and CTM. These are the overlays (serviceand management-aware functions, also used for the interconnection with applications/services), the middleware and the underlays (Software Defined Radio underlay, virtualization functions like virtual Base Station and virtual Gateway, IP/MPLS, for interconnection with the networking elements). The convergence between wired and wireless networks as well as among heterogeneous wireless access technologies can be provided as well. In other words, user-centric communication can be offered for a multitude of services with the associated elements (network selection, seamless mobility, application and session management, safety and security provisions as well as guaranteed QoS/QoE). Other elements are in the form of cognitive network operation and management (CNOM) and the provision of advanced services like, e.g., energy efficiency. Cognitive networking means that networking entities (e.g., Base Stations, Mobile Terminals) have the ability to operate in a carrier independent way and to optimally take decisions regarding the selection of appropriate radio interfaces, transmit power level, MAC, routing protocol, e2e route as well as to organize into resource-optimized ad-hoc networks. The wireless cloud also offers scalability advantages like the possibility for link aggregation and radio selection over multiple operators. New RATs, RANs and operators can be added as well.

\section{CONCLUSIONS}

The paper has provided a short overview of the important problem of greening of IP-based video distribution networks. Video based services are expected to show a huge development in the future and, associated with this, they are associated with large energy consumption. Some of the most important technical challenges related to this have been investigated as well. They are associated with the greening of radio access networks and core networks, network virtualization, greening of content distribution and data centers as well as cognitive network operation and management. Solving these challenges demands for sustained scientific and monetary efforts. Altogether, there is high expectation that new technical solutions will appear to allow the advance and the acceptance of the new technology.

\section{REFERENCES}

[1] IP/MPLS Networks: Optimize Video Transport for Service Providers, CISCO Report, http://www.cisco.com/en/US/solutions/collateral/ns341/ ns 1043/ns/1106/ns1110/white_paper_c11-637031.pdf

[2] Hossain E., Bhargava V.K. and Fettweis G.P. (editors), Green Radio Communication Networks, Cambridge University Press, 2012

[3] ETSI Technical Specification TS 102706, Environment Engineering (EE) Energy Efficiency of Wireless Access Network, Reference DTS/EE 00007, Sophia Antipolis, France, http://www.etsi.org/deliver/etsi_ts/ 102700_102799/102706/01.01.01_60/ts_102706v010101p.pdf
[4] Haesik K., personal communications, VTT, Oulu, Finland, 2012

[5] Bianzino A.P., Chauder C., Rossi D. and Rougier J-L., A Survey of Green Networking Research, IEEE Communications Surveys \& Tutorials, Vol. 14, No. 2, Second Quarter 2012

[6] Hasan Z., Boostanimerh H. and Bhargava V.K., Green Cellular Networks: A Survey, Some Research Issues and Challenges, IEEE Communications Surveys \& Tutorials, Vol. 13, No. 4, Fourth Quarter 2011

[7] Lei L and Zhong Z., Challenges on Wireless Heterogeneous Networks for Mobile Cloud Computing, IEEE Wireless Communications, June 2013

[8] Guvenc I., Quek T.Q.S., Kountouris M. and Lopez-Perez D., (guest editors), Heterogeneous and Small Cell Networks: Part 2, IEEE Communications Magazine, Vol. 51, No. 6, June 2013

[9] Lane K., Backend as a Service (BaaS) Ecosystem, White Paper, Kinvey, May 2013

[10] Bolla R., Bruschi R., Davoli F. and Cucchietti F., Energy Efficiency in the Future Internet: A Survey of Existing Approaches and Trends in EnergyAware Fixed Network Infrastructures, IEEE Communications Surveys \& Tutorials, Vol. 13, No. 2, Second Quarter 2011

[11] Qureshi A., Weber R., Balakrishnan H., Guttag J. and Maggs B., Cutting the Electric Bill for Internet-Scale Systems, SIGCOMM 2009, Barcelona, Spain, September 2009

[12] Cianfrani A., Eramo V., Listanti M., Polverini M., An OSPF-Integrated Routing Strategy for QoS-Aware Energy Saving in IP Backbone Networks, IEEE Transactions on Network and Service Management, Vol. 9, No. 3, September 2012

[13] Nakao A., Aoyama T., Takahara N., Takahashi N., Tanaka H., Ogaki K., Hayashi M., Kuri T., Nakauchi K., Nishihara N., Suzuki T., Nishihara M., Kiriha Y., Matsubara D. and Ishihara T., Advanced Network Virtualization: Definition, Benefits, Applications, and Technical Challenges, White Paper, Version 1.0, January 2012

[14] Estevez R.P. and Granville L.Z., On the Management of Virtual Networks, IEEE Communications Magazine, July 2013

[15] Nakao A., Network Virtualization Enabling Deeply Programmable Network, University of Tokio, Japan, talk 2012/4/19

[16] DeCusatis C.J., Carranza A. and DeCusatis C.M., Communication Within Clouds: Open Standards and Proprietary Protocols for Data Center, IEEE Communications Magazine, September 2012

[17] Software Defined Networking: the New Norm for Networks, White Paper, Open Networking Foundation, April 2012, http://www.opennetworking.org/images/stories/downloads/whitepapers/wp-sdn-newnorm.pdf

[18] Mishra A., guest editor, Cloud Networking and Communications, IEEE Communications Magazine, November 2013

[19] 3GPP TS 32.500, Telecommunication Management; Self-Organizing Networks (SON): Concepts and Requirements (Rel 8), 3GPP documentation, December 2008

[20] Munoz L. (chair), Pampu C. (co-chair), Future Networks and Management, Net!Works White Paper, June 2011

[21] Sezer S., Scott-Hayward S., Chouhan P.K., Fraser B., Lake D., Finnegan J., Viljoen N., Miller M. and Rao N., Are We Ready for SDN? Implementation Challenges for Software-Defined Networks, IEEE Communications Magazine, July 2013

[22] Infrastructure to Application Information Exposure and Communications - DC Case, IETF i2aex BoF, draft version v0.6, March 26, 2012, http://www.ietf.org/proceedings/83/slides/slides-83-i2aex-2.pdf

[23] Pentikousis K., Wang Y. and Hu W., MobileFlow: Toward Software Defined Mobile Networks, IEEE Communications Magazine, July 2013

[24] Al-Mukaddim K.P., Buyya R. and Vakali A., Content Delivery Networks: State of the Art, Insights, and Imperatives, Content Delivery Networks, Lecture Notes, Springer, Electrical Engineering, Volume 9 , pp. 3-32, 2008

[25] Manzalini A. and Minerva R., Clouds of Virtual Machines in Edge Networks, IEEE Communications Magazine, July 2013

[26] Islam S. and Gregoire J-C., Giving Users an Edge: A Flexible Cloud Model and Its Application for Multimedia, journal of Future Generation Computer Systems, 28 (2012), pp. 823 - 832

[27] Nicolaou A., Best Practices on the Move: Building Web Apps for Mobile Devices, Communications of the ACM, Vol. 56, No. 8, August 2013

[28] Han T., Ansari N., Wu M. and Yu H., On Accelerating Content Delivery in Mobile Networks, IEEE Communications Surveys \& Tutorials, Vol. 15, No. 3, Third Quarter 2013 
[29] Bari M.F., Boutaba R., Esterves R., Zambenedetti L., Podlesny M., Rabbani M.G., Zhang Q. and Zhani M.F., Data Center Network Virtualization: A Survey, IEEE Communications Surveys \& Tutorials, Vol. 15, No. 2, Second Quarter 2013

[30] Tyson G., Mauthe A., Kaune S., Grace P., Tawelel A. and Plagemann T., Juno: A Middleware Platform for Supporting Delivery-Centric Application, ACM Transactions on Internet Technology, Vol. 12, No. 2, December 2012

[31] Popescu A., Haesik K., Davoli F., Lopez R.D., Thao S., Del Ser Lorente J. and Wunder G., ENVIRAN: Energy Efficient Radio Access Networks, Global Wireless Summit 2013, Atlantic City, New Jersey, USA, June 2013 